\title{
新型含取代吡啶结构的氭基丙烯酸酯类化合物的合成与除草活性研究
}

\author{
戴 红 ${ }^{a}$ 陈 佳 ${ }^{a}$ 洪宇 ${ }^{a}$ 袁斌颖 ${ }^{a}$ 陈雨蒙 ${ }^{a}$ \\ 石玉军*, $a$ 马瑞媛 ${ }^{a}$ 梁志鹏 $b$ 石 健*, $b$ \\ ( ${ }^{a}$ 南通大学化学化工学院 南通 226019) \\ ( ${ }^{b}$ 南通大学分析测试中心 南通 226019)
}

\begin{abstract}
摘要 为了从氧基丙烯酸酯类化合物中寻找新的活性物质, 运用活性亚结构拼接方法, 设计并合成了一系列未见文献 报道的新型含取代吡啶结构的氧基丙烯酸酯类衍生物. 通过 ${ }^{1} \mathrm{H} N \mathrm{NR},{ }^{13} \mathrm{C} N \mathrm{NR}$ 和元素分析确认了目标化合物的结构. 初步的生物活性测试结果表明, 在测试浓度为 $1500 \mathrm{~g} \cdot \mathrm{ha}^{-1}$ 时, 2-氧基-3-甲硫基-3-[4-(5-三氟甲基吡啶-2-胺基)]苯甲基胺 基丙烯酸 (2-甲氧基)乙酯(7I)对芥菜的茎叶处理除草活性为 95\%, 2-氰基-3-甲硫基-3-[4-(5-三氟甲基吡啶-2-胺基)]苯甲基 胺基丙烯酸 2 - - (2,4-二氟苯氧基)]乙酯(7e), 71 和 2-萻基-3-甲硫基-3-[4-(5-三氟甲基吡啶-2-胺基)]苯甲胺基丙烯酸(2-乙氧 基)乙酯(7m) 对繁缕的茎叶处理除草活性分别为 80\%，80\%和 100\%, 此外, 2-氰基-3-甲硫基-3-[4-(5-三氟甲基吡啶-2-胺 基)]苯甲基胺基丙烯酸 $[2$-(2-氟苯氧基)]乙酯(7b), 71 和 7m 对小菉的茎叶处理除草活性均达 $100 \%$.
\end{abstract}

关键词＼cjkstart吡啶；萻基丙烯酸酯; 合成; 除草活性

\section{Synthesis and Herbicidal Activity of Novel Cyanoacrylates Containing Substituted Pyridyl Moiety}

\author{
Dai, Hong ${ }^{a}$ \\ Chen, $\mathrm{Jia}^{a}$ \\ Hong, $\mathrm{Yu}^{a}$ \\ Yuan, Binying ${ }^{a}$ \\ Chen, Yumeng ${ }^{a}$ \\ Shi, Yujun ${ }^{*, a} \quad$ Ma, Ruiyuan ${ }^{a} \quad$ Liang, Zhipeng $^{b} \quad$ Shi, Jian*,b \\ $\left({ }^{a}\right.$ College of Chemistry and Chemical Engineering, Nantong University, Nantong 226019) \\ ( ${ }^{b}$ Analysis and Testing Center, Nantong University, Nantong 226019)
}

\begin{abstract}
In order to find new cyanoacrylate lead compounds, a series of novel cyanoacrylates containing substituted pyridyl moiety were prepared by the method of active substructure combination. The structures of the target compounds were confirmed by ${ }^{1} \mathrm{H}$ NMR, ${ }^{13} \mathrm{C}$ NMR and elemental analyses. Preliminary bioassay data displayed that in postemergence treatment 2-cyano-3-methylthio-3-[4-(5-trifluoromethylpyridyl-2-amino)]benzylamino(2-methoxy)ethyl ester (7l) had 95\% herbicidal activity against Brassica juncea at $1500 \mathrm{~g} / \mathrm{ha}$ and 2-cyano-3-methylthio-3-[4-(5-trifluoromethylpyridyl-2-amino)]benzylamino[2-(2,4-difluorophenoxy)]ethyl ester (7e), 71 and 2-cyano-3-methylthio-3-[4-(5-trifluoromethylpyridyl-2-amino)]benzylamino(2-ethoxy)ethyl ester $(7 \mathbf{m})$ showed $80 \%, 80 \%$ and $100 \%$ herbicidal activity against Stellaria media, respectively. Additionally, 2-cyano-3-methylthio-3-[4-(5-trifluoromethylpyridyl-2-amino)]benzylamino[2-(2-fluorophenoxy)]ethyl ester (7b), $7 \mathbf{l}$ and $7 \mathbf{m}$ all exhibited $100 \%$ herbicidal activity against Chenopodium serotinum $L$. at $1500 \mathrm{~g} / \mathrm{ha}$.
\end{abstract}

Keywords pyridine; cyanoacrylate; synthesis; herbicidal activity

氧基丙烯酸酯类化合物是一类活性较高的光系统 II (PSII)电子传递抑制剂，因其具有独特的作用机制、较高 的选择性及较好的环境相容性等特点而倍受广大药物 化学工作者的关注. 在近 10 多年的研究中多种活性的 氧基丙烯酸酯类化合物被成功开发出来, 在生物学方面
具有广泛的应用 ${ }^{[1 \sim 5]}$. 如 Sun 等 ${ }^{[6]}$ 报道的含二茂铁结构 的氰基丙烯酸酯类衍生物表现出较好的除草作用, Song 等 ${ }^{[7]}$ 合成的含取代苯基结构的氰基丙烯酸酯化合物呈现 优异的抗植物病毒活性, Liu 等 ${ }^{[8]}$ 合成的含呋喃、四氢呋 喃环的氰基丙烯酸酯类化合物具有良好的杀菌和除草

* Corresponding authors. E-mail: yjshi2015@163.com, gaohbhe2015@aliyun.com

Received November 23, 2016; revised December 8, 2016; published online December 21, 2016.

Project supported by the National Natural Science Foundation of China (No. 21372135), the Research Foundation of the Six People Peak of Jiangsu Province (No. 2013-SWYY-013) and the Science and Technology Innovation Foundation for the College Students of Jiangsu Province (No. 201610304047Z).

国家自然科学基金(No. 21372135)、江苏省 “六大人才高峰” (No. 2013-SWYY-013)和江苏省大学生创新训练计划(No. 201610304047Z)资助项目. 
活性, Zhao 等 ${ }^{[9]}$ 报道的含噁唑、噁二唑结构的氰基丙烯 酸酯衍生物表现出优良的除草与植物生长调节活性. 另 外, 吡定类化合物作为含氮杂环中的重要一员, 具有杀 虫、杀菌、除草、植物生长调节及抗病毒等活性 ${ }^{[10 ~ 14]}$, 在 农业生产中发挥着举足轻重的作用, 如杀菌剂啶斑肟、 氟啶胺; 杀虫剂氯虫苯甲酰胺、吡虫啉; 除草剂吡氟氯 禾灵和吡氟禾草灵等 ${ }^{[15 ~ 19] . ~}$. 为了进一步从氧基丙烯酸 酯类化合物中寻找高活性的先导化合物, 本工作采用活 性基团拼接原理, 将取代吡啶结构单元引入到氰基丙烯 酸酯分子结构中, 设计合成了一系列未见文献报道的新 型含取代吡定结构的氰基丙烯酸酯类衍生物. 通过核磁 氢谱、碳谱和元素分析等方法确证了目标化合物的结构. 初步的除草活性测试结果显示, 部分目标物表现出较好 的除草活性. 其合成路线如 Scheme 1 所示.

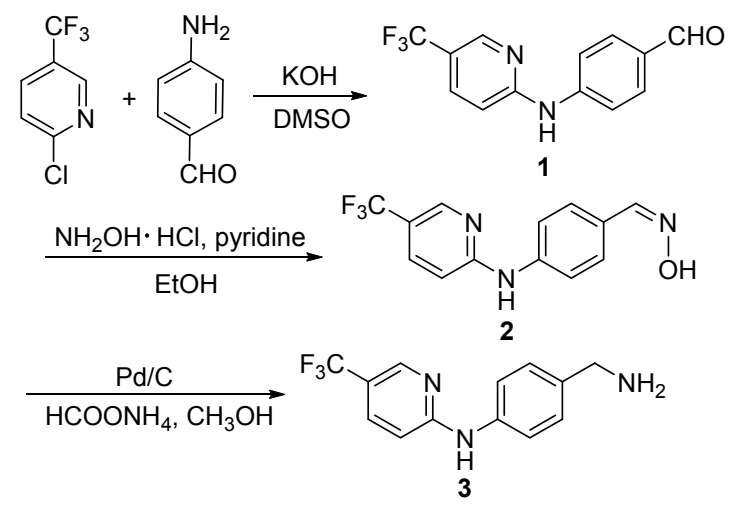

$\mathrm{CNCH}_{2} \mathrm{COOH}+\underset{4}{\mathrm{HOC}_{2} \mathrm{H}_{4} \mathrm{OR}^{2}} \underset{\text { or } \mathrm{H}_{2} \mathrm{SO}_{4}}{\stackrel{\mathrm{NaHSO}_{4}}{\longrightarrow}} \underset{\mathbf{5}}{\mathrm{CNCH}_{2} \mathrm{COOC}_{2} \mathrm{H}_{4} \mathrm{OR}^{2}}$

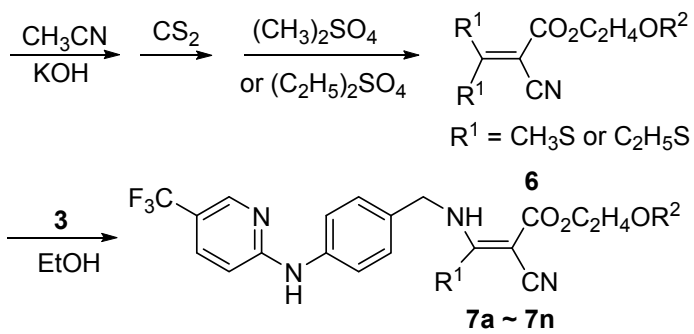

7a: $\mathrm{R}^{1}=\mathrm{SCH}_{3}, \mathrm{R}^{2}=4-\mathrm{CH}_{3} \mathrm{OC}_{6} \mathrm{H}_{4} ; 7 \mathbf{b}: \mathrm{R}^{1}=\mathrm{SCH}_{3}, \mathrm{R}^{2}=2-\mathrm{FC}_{6} \mathrm{H}_{4}$;

7c: $R^{1}=\mathrm{SCH}_{3}, \mathrm{R}^{2}=4-\mathrm{FC}_{6} \mathrm{H}_{4} ; 7 \mathrm{~d}: \mathrm{R}^{1}=\mathrm{SCH}_{3}, \mathrm{R}^{2}=2,3-\mathrm{F}_{2} \mathrm{C}_{6} \mathrm{H}_{3}$;

7e: $\mathrm{R}^{1}=\mathrm{SCH}_{3}, \mathrm{R}^{2}=2,4-\mathrm{F}_{2} \mathrm{C}_{6} \mathrm{H}_{3} ; 7 \mathrm{ff}: \mathrm{R}^{1}=\mathrm{SCH}_{3}, \mathrm{R}^{2}=3,4-\mathrm{F}_{2} \mathrm{C}_{6} \mathrm{H}_{3}$;

7g: $\mathrm{R}^{1}=\mathrm{SCH}_{3}, \mathrm{R}^{2}=3,5-\mathrm{F}_{2} \mathrm{C}_{6} \mathrm{H}_{3} ; \mathbf{7 h}: \mathrm{R}^{1}=\mathrm{SCH}_{3}, \mathrm{R}^{2}=2,3-\mathrm{Cl}_{2} \mathrm{C}_{6} \mathrm{H}_{3}$;

7i: $\mathrm{R}^{1}=\mathrm{SCH}_{3}, \mathrm{R}^{2}=2,4-\mathrm{Cl}_{2} \mathrm{C}_{6} \mathrm{H}_{3} ; 7 \mathrm{j}: \mathrm{R}^{1}=\mathrm{SCH}_{3}, \mathrm{R}^{2}=3,4-\mathrm{Cl}_{2} \mathrm{C}_{6} \mathrm{H}_{3}$;

7k: $\mathrm{R}^{1}=\mathrm{SCH}_{3}, \mathrm{R}^{2}=4-\mathrm{BrC}_{6} \mathrm{H}_{4} ;$ 7l: $\mathrm{R}^{1}=\mathrm{SCH}_{3}, \mathrm{R}^{2}=\mathrm{CH}_{3}$;

$7 m: R^{1}=S H_{3}, R^{2}=C_{2} H_{5} ; 7 n: R^{1}=S_{2} H_{5}, R^{2}=4-F_{6} H_{4}$

图式 1 目标化合物 7 的合成路线

Scheme 1 Synthetic route of target compounds 7

\section{1 结果与讨论}

\section{1 化合物的合成}

关键中间体 1 是通过 2-氯-5-三氟甲基吡啶与 4-氨 基苯甲醛的缩合反应制备而成. 试探了不同的反应条件
对其反应收率的影响. 由表 1 可以看出, 采用 $\mathrm{KOH}$ 作缚 酸剂，DMSO 作溶剂，室温反应 $12 \mathrm{~h}$ ，是合成关键中间 体 1 的较佳方法，中间体 1 的收率为 $52 \%$.

在目标化合物 7 的合成过程中，我们以化合物 7a 的合成为研究对象，探讨了不同的溶剂(如甲醇、乙醇、 乙腈及 $N, N$-二甲基甲酰胺等)及反应温度对化合物 7a 收 率的影响. 通过研究发现, 选用乙醇作溶剂, 加热回流 的方法，不仅能使反应顺利进行，而且后处理也较为简 便，化合物 7a 的反应收率为 $61 \%$. 因此，采用该方法成 功地制备了其它的含取代吡啶结构的氰基丙烯酸酯类 化合物.

\section{2 化合物的图谱分析}

以目标化合物 7a 的核磁氢谱和核磁碳谱数据为例 进行说明. 在 $\delta 10.19$ 处的单峰为 $\mathrm{CH}_{2} \mathrm{NH}$ 上胺基氢的吸 收峰, $\delta 9.67$ 处的单峰为另一个胺基氢的吸收峰, $\delta 8.48$ 处的单峰为吡啶环上一个氢的吸收峰， $\delta 7.85 \sim 7.87$ 间 的多重峰为吡啶环上一个氢的吸收峰, $\delta 7.71$ 处的双重 峰为苯环上两个氢的吸收峰, $\delta 7.27$ 处的双重峰为苯环 上两个氢的吸收峰, $\delta 6.83 \sim 6.96$ 间的多重峰为苯环上 四个氢和吡啶环上一个氢的吸收峰, $\delta 4.75$ 处的单峰为 $\mathrm{NCH}_{2}$ 上亚甲基两个氢的吸收峰, $\delta 4.40$ 处的三重峰为与 酯基相连的亚甲基上两个氢的吸收峰, $\delta 4.14$ 处的三重 峰为与苯氧基相连的亚甲基上两个氢的吸收峰， $\delta 3.69$ 处的单峰为苯环 4-位甲氧基上三个氢的吸收峰, $\delta 2.62$ 处的单峰为甲硫基上三个氢的吸收峰, $\delta$ 在 66.8 处的峰 为与苯环相连的 $\mathrm{OCH}_{2}$ 碳原子的信号峰, $\delta 63.1$ 处的峰为 与羰基相连的 $\mathrm{OCH}_{2}$ 碳原子的信号峰, $\delta 55.7$ 处的峰为与 苯环相连的 $\mathrm{OCH}_{3}$ 碳原子的信号峰, $\delta 49.1$ 处的峰为 $\mathrm{NCH}_{2}$ 碳原子的信号峰, $\delta 18.3$ 处的峰为 $\mathrm{SCH}_{3}$ 碳原子的 信号峰.

\section{3 化合物的除草活性}

目标化合物的除草活性测试结果如表 2 所示. 初步 的试验结果表明, 在 $1500 \mathrm{~g} / \mathrm{ha}$ 浓度下, 采用土壤处理 时, 所有化合物对芥菜(Brassica juncea)、繁缕(Stellaria media)和小㢣(Chenopodium serotinum L.)均未显示除草 效果; 当采用茎叶处理时, 部分目标物呈现较好的除草 活性，但无明显的规律. 化合物 71 对芥菜的茎叶处理抑 制活性达到 95\%，与对照药 NK-9717 的除草效果相近; 化合与对照药 NK-9717 的除草效果相近; 化合物 7e, 71 和 $7 \mathrm{~m}$ 对繁缕的茎叶处理抑制活性分别为 $80 \%, 80 \%$ 和 $100 \%$ ，其中化合物 7m 与对照药 NK-9717 的除草活性相 当; 此外，化合物 $7 \mathrm{~b}, 71$ 和 $7 \mathrm{~m}$ 对小藜的茎叶处理抑制活 性均达 100\%，与对照药 NK-9717 的除草活性相当. 从 表 2 可以看出，当 $\mathrm{R}^{1}=\mathrm{SCH}_{3}$ 时，烷基化合物 71 和 $7 \mathbf{m}$ 对 
表 1 不同反应条件对中间体 1 合成收率的影响

Table 1 Effects of reaction conditions on the synthesis of the intermediate 1

\begin{tabular}{ccclc}
\hline Entry & Acid-binding agent & Solvent & \multicolumn{1}{c}{ Reaction condition } & Yield/\% \\
\hline 1 & $\mathrm{~K}_{2} \mathrm{CO}_{3}$ & $\mathrm{CH}_{3} \mathrm{CN}$ & Room temperature for $12 \mathrm{~h}$ & 0 \\
2 & $\mathrm{~K}_{2} \mathrm{CO}_{3}$ & $\mathrm{CH}_{3} \mathrm{CN}$ & Reflux for 12 $\mathrm{h}$ & 0 \\
3 & $\mathrm{NaOH}$ & $\mathrm{DMF}$ & Room temperature for $12 \mathrm{~h}$ & 0 \\
4 & $\mathrm{KOH}$ & $\mathrm{DMF}$ & Room temperature for $12 \mathrm{~h}$ & 0 \\
5 & $\mathrm{KOH}$ & $\mathrm{DMSO}$ & Room temperature for $12 \mathrm{~h}$ & 52 \\
\hline
\end{tabular}

表 2 目标化合物 $7 \mathbf{a} \sim 7 \mathbf{n}$ 的除草活性(抑制率/\%)

Table 2 Herbicidal activities of target compounds $7 \mathbf{a} \sim 7 \mathbf{n}$ (percent inhibition/\%)

\begin{tabular}{|c|c|c|c|c|c|c|c|}
\hline \multirow[b]{2}{*}{ Compd. } & \multirow[b]{2}{*}{ Dosage $/\left(\mathrm{g} \bullet \mathrm{ha}^{-1}\right)$} & \multicolumn{2}{|c|}{ Brassica juncea } & \multicolumn{2}{|c|}{ Stellaria media } & \multicolumn{2}{|c|}{ Chenopodium serotinum $L$. } \\
\hline & & $\begin{array}{l}\text { Pre-emergence } \\
\text { treatment }\end{array}$ & $\begin{array}{l}\text { Post emergence } \\
\text { treatment }\end{array}$ & $\begin{array}{l}\text { Pre-emergence } \\
\text { treatment }\end{array}$ & $\begin{array}{c}\text { Post emergence } \\
\text { treatment }\end{array}$ & $\begin{array}{l}\text { Pre-emergence } \\
\text { treatment }\end{array}$ & $\begin{array}{c}\text { Post emergence } \\
\text { treatment }\end{array}$ \\
\hline $7 a$ & 1500 & 0 & 0 & 0 & 60 & 0 & 50 \\
\hline $7 b$ & 1500 & 0 & 0 & 0 & 60 & 0 & 100 \\
\hline $7 \mathrm{c}$ & 1500 & 0 & 0 & 0 & 30 & 0 & 30 \\
\hline 7d & 1500 & 0 & 0 & 0 & 20 & 0 & 20 \\
\hline $7 e$ & 1500 & 0 & 0 & 0 & 80 & 0 & 50 \\
\hline $7 f$ & 1500 & 0 & 0 & 0 & 60 & 0 & 60 \\
\hline $7 \mathrm{~g}$ & 1500 & 0 & 0 & 0 & 20 & 0 & 40 \\
\hline $7 \mathrm{~h}$ & 1500 & 0 & 0 & 0 & 40 & 0 & 0 \\
\hline $7 \mathbf{i}$ & 1500 & 0 & 0 & 0 & 40 & 0 & 10 \\
\hline $7 \mathbf{j}$ & 1500 & 0 & 0 & 0 & 30 & 0 & 0 \\
\hline $7 k$ & 1500 & 0 & 0 & 0 & 40 & 0 & 50 \\
\hline 71 & 1500 & 0 & 95 & 0 & 80 & 0 & 100 \\
\hline $7 \mathrm{~m}$ & 1500 & 0 & 0 & 0 & 100 & 0 & 100 \\
\hline $7 n$ & 1500 & 0 & 0 & 0 & 30 & 0 & 40 \\
\hline NK-9717 & 1500 & 0 & 100 & 0 & 100 & 0 & 100 \\
\hline
\end{tabular}

繁缕和小藜的茎叶处理抑制效果相对较好. 进一步的结 构衍生与除草活性研究正在进行中.

\section{2 结论}

利用活性亚结构拼接原理，设计并制备了 14 个新 型含取代吡啶环结构的氰基丙烯酸酯类化合物. 初步的 生测结果表明，在 $1500 \mathrm{~g} \bullet \mathrm{ha}^{-1}$ 测试剂量下，化合物 71 对 芥菜的茎叶处理除草效果较好, 抑制率为 $95 \%$; 化合物 7e, 71 和 $7 \mathrm{~m}$ 对繁缕的茎叶处理除草活性相对较高, 抑制 率分别为 $80 \%, 80 \%$ 和 $100 \%$; 此外, 化合物 $7 \mathbf{b}, 7 \mathbf{1}$ 和 $7 \mathbf{m}$ 对小藜的茎叶处理除草活性均为 $100 \%$, 与对照药 NK9717 的除草活性相当, 具有进一步研究的价值.

\section{3 实验部分}

\section{1 仪器与试剂}

X-4 型数字显示熔点测定仪(北京泰克仪器有限公 司), 温度计未经校正; Yanaco-CHN CORDER MT-3 自 动元素分析仪; Bruker AM-400 型核磁共振仪, 以 $\mathrm{CDCl}_{3}$ 或 DMSO- $d_{6}$ 为溶剂, TMS 为内标; 柱层析硅胶为 $\mathrm{H}$ 型(青岛海洋化工厂，200３00 目). 所用试剂均为分 析纯. 中间体氰基乙酸-2-甲(乙或取代苯)氧基乙酯(5)和
2-氧基-3,3-二甲(乙)硫基丙烯酸取代酯(6)参照文献[20] 方法合成.

\subsection{4-(5-三氟甲基吡啶-2-胺基)苯甲醛(1)的合成}

在 $100 \mathrm{~mL}$ 圆底烧瓶中加入 $0.02 \mathrm{~mol} 2$-氯-5-三氟甲 基吡啶、 $0.02 \mathrm{~mol}$ 对氨基苯甲醛和 $40 \mathrm{~mL}$ 二甲亚砜 (DMSO), 室温摚拌下, 向其中加入 $0.04 \mathrm{~mol} 85 \%$ 的氢 氧化钾, 加毕, 继续室温搅拌 $12 \mathrm{~h}$. 停止反应, 将反应 液缓慢倒入盛有 $100 \mathrm{~mL}$ 水的烧杯中, 乙酸乙酯萃取数 次, 有机相用无水硫酸镁干燥, 过滤, 浓缩得到关键中 间体 1, 产品可直接用于后面的反应.

\subsection{4-(5-三氟甲基吡啶-2-胺基)苯甲醛肜(2)的合成}

在 $100 \mathrm{~mL}$ 圆底烧瓶中加入 $0.02 \mathrm{~mol}$ 关键中间体 $\mathbf{1}$ 、 $0.03 \mathrm{~mol}$ 盐酸差胺及 $25 \mathrm{~mL}$ 无水乙醇, 室温搅拌下, 向 其中加入 $6 \mathrm{~mL}$ 吡啶, 加毕, 升温回流反应 $8 \mathrm{~h}$. 停止反 应, 将反应液缓慢倒入盛有 $100 \mathrm{~mL}$ 水的烧杯中，乙酸 乙酯萃取数次, 有机相用无水硫酸镁干燥, 过滤, 浓缩 得到关键中间体 2 , 产品可直接用于后面的反应.

\subsection{4-(5-三氟甲基吡啶-2-胺基)苯甲胺(3)的合成}

在 $100 \mathrm{~mL}$ 圆底烧瓶中加入 $0.02 \mathrm{~mol}$ 关键中间体 2 、 $0.1 \mathrm{~mol}$ 甲酸铵及 $40 \mathrm{~mL}$ 甲醇, 室温搅拌下, 向其中加入 
适量的 $10 \% \mathrm{Pd} / \mathrm{C}$ 钯碳, 加毕, 升温回流反应 $10 \mathrm{~h}$. 停止 反应，抽滤，向滤液中加入 $30 \mathrm{~mL}$ 水，乙醚萃取数次， 有机相用无水硫酸镁干燥, 过滤, 浓缩得到关键中间体 3, 产品不经纯化直接用于下一步反应.

\section{5 目标化合物 7 的合成}

在 $100 \mathrm{~mL}$ 圆底烧瓶中, 加入 $3 \mathrm{mmol}$ 中间体 3、3 mmol 2-氧基-3,3-二甲(乙)硫基丙烯酸取代酯 6 及 $20 \mathrm{~mL}$ 无水乙醇. 将反应液加热回流, 薄层色谱(TLC)跟踪. 反 应结束后, 向反应液中加入 $30 \mathrm{~mL}$ 水, 乙酸乙酯萃取数 次, 有机层用无水硫酸钠干燥, 抽滤, 脱溶, 所得残余 物经柱层析 $[V$ (石油醚) $: V($ 乙酸乙酯 $)=1: 1]$ 分离得到 目标化合物 $7 \mathbf{a} \sim 7 \mathbf{n}$.

2-氧基-3-甲硫基-3-[4-(5-三氟甲基吡啶-2-胺基)]苯 甲基胺基丙烯酸 $[2-$ (4-甲氧基苯氧基)]乙酯(7a): 黄色固 体, 产率 61\%. m.p. 101 102 ${ }^{\circ} \mathrm{C}$; ${ }^{1} \mathrm{H}$ NMR $(400 \mathrm{MHz}$, DMSO- $\left.d_{6}\right) \delta: 10.19(\mathrm{~s}, 1 \mathrm{H}, \mathrm{NH}), 9.67(\mathrm{~s}, 1 \mathrm{H}, \mathrm{NH}), 8.48(\mathrm{~s}$, $1 \mathrm{H}$, Py-H), $7.85 \sim 7.87$ (m, 1H, Py-H), 7.71 (d, $J=12.0$ $\mathrm{Hz}, 2 \mathrm{H}, \mathrm{Ar}-\mathrm{H}), 7.27$ (d, $J=8.0 \mathrm{~Hz}, 2 \mathrm{H}, \mathrm{Ar}-\mathrm{H}), 6.83 \sim 6.96$ (m, 5H, Ar-H and Py-H), 4.75 (s, 2H, $\mathrm{CH}_{2}$ ), 4.40 (t, $J=4.0$ $\left.\mathrm{Hz}, 2 \mathrm{H}, \mathrm{CH}_{2}\right), 4.14\left(\mathrm{t}, J=4.0 \mathrm{~Hz}, 2 \mathrm{H}, \mathrm{CH}_{2}\right), 3.69(\mathrm{~s}, 3 \mathrm{H}$, $\left.\mathrm{OCH}_{3}\right), 2.62$ (s, 3H, $\left.\mathrm{SCH}_{3}\right) ;{ }^{13} \mathrm{C} \mathrm{NMR}\left(100 \mathrm{MHz}, \mathrm{CDCl}_{3}\right)$ $\delta: 172.8,168.2,157.8,154.2,152.6,146.0,139.2,134.9$, $131.6,128.4,125.5,122.8,121.4,118.2,118.0,117.6$, 116.0, 114.6, 107.9, 74.8, 66.8, 63.1, 55.7, 49.1, 18.3. Anal. calcd for $\mathrm{C}_{27} \mathrm{H}_{25} \mathrm{~F}_{3} \mathrm{~N}_{4} \mathrm{O}_{4} \mathrm{~S}$ : C 58.06, H 4.51, N 10.03; found C 58.17, H 4.39, N 10.12 .

2-氧基-3-甲硫基-3-[4-(5-三氟甲基吡啶-2-胺基)]苯 甲基胺基丙烯酸 $[2-(2$-氟苯氧基)]乙酯(7b): 黄色固体, 产率 58\%. m.p. $110 \sim 112{ }^{\circ} \mathrm{C} ;{ }^{1} \mathrm{H}$ NMR $(400 \mathrm{MHz}$, $\left.\mathrm{CDCl}_{3}\right) \delta: 10.32(\mathrm{~s}, 1 \mathrm{H}, \mathrm{NH}), 8.46(\mathrm{~s}, 1 \mathrm{H}, \mathrm{Py}-\mathrm{H}), 7.67 \sim$ $7.70(\mathrm{~m}, 1 \mathrm{H}, \mathrm{Py}-\mathrm{H}), 7.45$ (d, J=8.0 Hz, 2H, Ar-H), 7.05 $7.28(\mathrm{~m}, 5 \mathrm{H}$, Ar- $\mathrm{H}), 6.91 \sim 6.96(\mathrm{~m}, 1 \mathrm{H}, \mathrm{Ar}-\mathrm{H}), 6.86(\mathrm{~d}$, $J=8.0 \mathrm{~Hz}, 1 \mathrm{H}, \mathrm{Py}-\mathrm{H}), 4.78\left(\mathrm{~d}, J=8.0 \mathrm{~Hz}, 2 \mathrm{H}, \mathrm{CH}_{2}\right), 4.53$ (t, $\left.J=6.0 \mathrm{~Hz}, 2 \mathrm{H}, \mathrm{CH}_{2}\right), 4.32\left(\mathrm{t}, J=6.0 \mathrm{~Hz}, 2 \mathrm{H}, \mathrm{CH}_{2}\right), 2.70$ (s, $\left.3 \mathrm{H}, \mathrm{SCH}_{3}\right) ;{ }^{13} \mathrm{C}$ NMR $\left(100 \mathrm{MHz}, \mathrm{CDCl}_{3}\right) \delta: 172.9$, $168.1,157.8,154.2,151.8,146.5,146.0,139.1,134.9$, $131.6,128.4,124.4,122.8,122.0,121.4,118.1,117.6$, $116.4,116.3,116.2,107.9,74.7,67.6,62.8,49.1,18.3$. Anal. calcd for $\mathrm{C}_{26} \mathrm{H}_{22} \mathrm{~F}_{4} \mathrm{~N}_{4} \mathrm{O}_{3} \mathrm{~S}$ : C 57.14, H 4.06, N 10.25; found C 57.03, H 4.19, N 10.38.

2-氰基-3-甲硫基-3-[4-(5-三氟甲基吡啶-2-胺基)]苯 甲基胺基丙烯酸 $[2-(4$-氟苯氧基)]乙酯(7c): 黄色固体, 产率 63\%. m.p. $106 \sim 108{ }^{\circ} \mathrm{C} ;{ }^{1} \mathrm{H}$ NMR $(400 \mathrm{MHz}$, $\left.\mathrm{CDCl}_{3}\right) \delta: 10.33$ (s, 1H, NH), 8.47 (s, 1H, Py-H), 7.68 7.71 (m, 1H, Py-H), 7.46 (d, $J=8.4$ Hz, 2H, Ar-H), 6.85 $7.28(\mathrm{~m}, 7 \mathrm{H}$, Ar-H and Py-H), $4.78(\mathrm{~d}, J=6.0 \mathrm{~Hz}, 2 \mathrm{H}$, $\left.\mathrm{CH}_{2}\right), 4.50\left(\mathrm{t}, \quad J=5.2 \mathrm{~Hz}, 2 \mathrm{H}, \mathrm{CH}_{2}\right), 4.20(\mathrm{t}, J=5.2 \mathrm{~Hz}$, $\left.2 \mathrm{H}, \mathrm{CH}_{2}\right), 2.71\left(\mathrm{~s}, 3 \mathrm{H}, \mathrm{SCH}_{3}\right) ;{ }^{13} \mathrm{C}$ NMR $(100 \mathrm{MHz}$, $\left.\mathrm{CDCl}_{3}\right) \delta: 172.9,168.2,158.7,157.7,156.3,154.6,146.0$, $139.2,134.9,131.5,128.5,125.5,122.8,122.5,121.4$, 118.1, 117.7, 116.0, 115.9, 115.7, 107.9, 74.7, 66.6, 62.9, 49.2, 18.3. Anal. calcd for $\mathrm{C}_{26} \mathrm{H}_{22} \mathrm{~F}_{4} \mathrm{~N}_{4} \mathrm{O}_{3} \mathrm{~S}$ : C 57.14, $\mathrm{H}$ 4.06, N 10.25; found C 57.26, H 3.95, N 10.13.

2-氰基-3-甲硫基-3-[4-(5-三氟甲基吡啶-2-胺基)]苯 甲基胺基丙烯酸 $[2-(2,3-$ 二氟苯氧基)]乙酯(7d): 灰色固 体, 产率 69\%. m.p. 120 122 ${ }^{\circ} \mathrm{C} ;{ }^{1} \mathrm{H}$ NMR $(400 \mathrm{MHz}$, DMSO- $\left.d_{6}\right) \delta: 10.18(\mathrm{~s}, 1 \mathrm{H}, \mathrm{NH}), 9.66(\mathrm{~s}, 1 \mathrm{H}, \mathrm{NH}), 8.48(\mathrm{~s}$, $1 \mathrm{H}, \mathrm{Py}-\mathrm{H}), 7.84 \sim 7.87$ (m, 1H, Py-H), 7.70 (d, $J=8.4 \mathrm{~Hz}$, 2H, Ar-H), 7.26 (d, J=8.4 Hz, 2H, Ar-H), $7.01 \sim 7.14$ (m, $3 \mathrm{H}$, Ar-H), 6.95 (d, $J=8.8 \mathrm{~Hz}, 1 \mathrm{H}, \mathrm{Py}-\mathrm{H}), 4.75$ (s, 2H, $\mathrm{CH}_{2}$ ), $4.46\left(\mathrm{t}, J=4.4 \mathrm{~Hz}, 2 \mathrm{H}, \mathrm{CH}_{2}\right), 4.34(\mathrm{t}, J=4.4 \mathrm{~Hz}, 2 \mathrm{H}$, $\left.\mathrm{CH}_{2}\right), 2.62\left(\mathrm{~s}, 3 \mathrm{H}, \mathrm{SCH}_{3}\right) ;{ }^{13} \mathrm{C} \mathrm{NMR}\left(100 \mathrm{MHz}, \mathrm{CDCl}_{3}\right) \delta$ : $172.9,168.0,157.7,152.7,150.3,148.1,145.9,143.0$, $140.5,139.1,135.0,131.6,128.5,125.5,123.4,121.5$, 118.1, 110.9, 110.0, 109.8, 107.9, 74.6, 67.9, 62.6, 49.2, 18.3. Anal. calcd for $\mathrm{C}_{26} \mathrm{H}_{21} \mathrm{~F}_{5} \mathrm{~N}_{4} \mathrm{O}_{3} \mathrm{~S}$ : C 55.32, H 3.75, N 9.92; found C 55.19, H 3.88, N 9.78.

2-氰基-3-甲硫基-3-[4-(5-三氟甲基吡啶-2-胺基)]苯 甲基胺基丙烯酸 $[2-(2,4-$ 二氟苯氧基) ]乙酯(7e): 灰色固 体, 产率 54\%. m.p. 131 133 ${ }^{\circ} \mathrm{C} ;{ }^{1} \mathrm{H}$ NMR $(400 \mathrm{MHz}$, DMSO- $\left.d_{6}\right) \delta: 10.19(\mathrm{~s}, 1 \mathrm{H}, \mathrm{NH}), 9.66(\mathrm{~s}, 1 \mathrm{H}, \mathrm{NH}), 8.48$ (s, $1 \mathrm{H}, \mathrm{Py}-\mathrm{H}$ ), $7.85 \sim 7.87$ (m, 1H, Py-H), 7.70 (d, $J=8.4 \mathrm{~Hz}$, $2 \mathrm{H}$, Ar-H), 6.94 $7.31(\mathrm{~m}, 6 \mathrm{H}$, Ar-H and Py-H), 4.76 (d, $\left.J=5.2 \mathrm{~Hz}, 2 \mathrm{H}, \mathrm{CH}_{2}\right), 4.43\left(\mathrm{t}, J=4.4 \mathrm{~Hz}, 2 \mathrm{H}, \mathrm{CH}_{2}\right), 4.28(\mathrm{t}$, $\left.J=4.4 \mathrm{~Hz}, 2 \mathrm{H}, \mathrm{CH}_{2}\right), 2.62\left(\mathrm{~s}, 3 \mathrm{H}, \mathrm{SCH}_{3}\right) ;{ }^{13} \mathrm{C} \mathrm{NMR}(100$ $\left.\mathrm{MHz}, \mathrm{CDCl}_{3}\right) \delta: 172.9,168.1,157.7,154.2,151.8,145.9$, $143.0,139.1,135.0,131.6,128.5,125.5,122.8,121.5$, 118.1, 117.7, 117.6, 110.7, 110.6, 108.0, 105.0, 74.7, 68.7, 62.9, 49.1, 18.3. Anal. calcd for $\mathrm{C}_{26} \mathrm{H}_{21} \mathrm{~F}_{5} \mathrm{~N}_{4} \mathrm{O}_{3} \mathrm{~S}$ : C 55.32, H 3.75, N 9.92; found C 55.46, H 3.62, N 9.80.

2-氧基-3-甲硫基-3-[4-(5-三氟甲基吡啶-2-胺基)]苯 甲基胺基丙烯酸 $[2-(3,4-$ 二氟苯氧基)]乙酯(7f)：灰色固 体, 产率 63\%. m.p. $148 \sim 150{ }^{\circ} \mathrm{C} ;{ }^{1} \mathrm{H}$ NMR $(400 \mathrm{MHz}$, DMSO- $\left._{6}\right) \delta: 10.18(\mathrm{~s}, 1 \mathrm{H}, \mathrm{NH}), 9.66(\mathrm{~s}, 1 \mathrm{H}, \mathrm{NH}), 8.48(\mathrm{~s}$, 1H, Py-H), 7.86 (d, $J=8.0 \mathrm{~Hz}, 1 \mathrm{H}, \mathrm{Py}-\mathrm{H}), 7.70$ (d, $J=8.0$ $\mathrm{Hz}, 2 \mathrm{H}, \mathrm{Ar}-\mathrm{H}$ ), $7.30 \sim 7.37$ (m, 1H, Ar-H), 7.26 (d, $J=8.0$ $\mathrm{Hz}, 2 \mathrm{H}, \mathrm{Ar}-\mathrm{H}$ ), $6.79 \sim 7.13$ (m, 3H, Ar-H and Py-H), 4.76 (d, $\left.J=4.0 \mathrm{~Hz}, 2 \mathrm{H}, \mathrm{CH}_{2}\right), 4.42\left(\mathrm{t}, J=6.0 \mathrm{~Hz}, 2 \mathrm{H}, \mathrm{CH}_{2}\right.$ ), $4.22\left(\mathrm{t}, \quad J=4.0 \mathrm{~Hz}, 2 \mathrm{H}, \mathrm{CH}_{2}\right), 2.62\left(\mathrm{~s}, 3 \mathrm{H}, \mathrm{SCH}_{3}\right) ;{ }^{13} \mathrm{C}$ NMR $\left(100 \mathrm{MHz}, \mathrm{CDCl}_{3}\right) \delta: 173.0,168.1,157.7,154.8$, 
$151.7,149.3,146.6,145.9,144.2,135.0,131.6,128.5$, $125.5,122.8,121.5,118.1,117.3,110.1,108.0,104.8$, 104.6, 74.6, 66.7, 62.7, 49.2, 18.3. Anal. calcd for $\mathrm{C}_{26} \mathrm{H}_{21}{ }^{-}$ $\mathrm{F}_{5} \mathrm{~N}_{4} \mathrm{O}_{3} \mathrm{~S}$ : C 55.32, H 3.75, N 9.92; found C 55.21, H 3.86, $\mathrm{N} 10.05$.

2-氰基-3-甲硫基-3-[4-(5-三氟甲基吡啶-2-胺基)]苯 甲基胺基丙烯酸 $[2-(3,5-$ 二氟苯氧基)]乙酯(7g): 白色固 体, 产率 66\%. m.p. $138 \sim 141{ }^{\circ} \mathrm{C} ;{ }^{1} \mathrm{H}$ NMR $(400 \mathrm{MHz}$, $\left.\mathrm{CDCl}_{3}\right) \delta: 10.23(\mathrm{~s}, 1 \mathrm{H}, \mathrm{NH}), 8.37(\mathrm{~s}, 1 \mathrm{H}, \mathrm{Py}-\mathrm{H}), 7.62 \sim$ $7.64(\mathrm{~m}, 1 \mathrm{H}, \mathrm{Py}-\mathrm{H}), 7.35$ (d, J=8.4 Hz, 2H, Ar-H), 7.13 $7.19(\mathrm{~m}, 2 \mathrm{H}, \mathrm{Ar}-\mathrm{H}), 6.79$ (d, $J=8.8 \mathrm{~Hz}, 1 \mathrm{H}, \mathrm{Py}-\mathrm{H}), 6.33 \sim$ $6.38(\mathrm{~m}, 3 \mathrm{H}, \mathrm{Ar}-\mathrm{H}), 4.70\left(\mathrm{~d}, J=6.0 \mathrm{~Hz}, 2 \mathrm{H}, \mathrm{CH}_{2}\right), 4.42(\mathrm{t}$, $\left.J=4.8 \mathrm{~Hz}, 2 \mathrm{H}, \mathrm{CH}_{2}\right), 4.11\left(\mathrm{t}, J=4.8 \mathrm{~Hz}, 2 \mathrm{H}, \mathrm{CH}_{2}\right), 2.63$ (s, $\left.3 \mathrm{H}, \mathrm{SCH}_{3}\right) ;{ }^{13} \mathrm{C} \mathrm{NMR}\left(100 \mathrm{MHz}, \mathrm{CDCl}_{3}\right) \delta: 173.0,168.1$, $164.9,162.5,160.3,157.5,145.4,138.9,135.3,131.9$, $128.5,125.4,122.7,121.7,118.0,108.1,98.7,98.4,96.8$, 74.6, 66.4, 62.4, 49.2, 18.4. Anal. calcd for $\mathrm{C}_{26} \mathrm{H}_{21} \mathrm{~F}_{5^{-}}$ $\mathrm{N}_{4} \mathrm{O}_{3} \mathrm{~S}$ : C 55.32, H 3.75, N 9.92; found C 55.45, H 3.62, N 10.03 .

2-氰基-3-甲硫基-3-[4-(5-三氟甲基吡啶-2-胺基)]苯 甲基胺基丙烯酸 $[2-(2,3-$ 二氯苯氧基)]乙酯(7h): 灰色固 体, 产率 62\%. m.p. 106 $108{ }^{\circ} \mathrm{C} ;{ }^{1} \mathrm{H}$ NMR (400 MHz, DMSO- $\left.d_{6}\right) \delta: 10.19(\mathrm{~s}, 1 \mathrm{H}, \mathrm{NH}), 9.66(\mathrm{~s}, 1 \mathrm{H}, \mathrm{NH}), 8.48$ (s, 1H, Py-H), $7.84 \sim 7.87$ (m, 1H, Py-H), 7.70 (d, $J=8.4$ Hz, 2H, Ar-H), $7.19 \sim 7.33(\mathrm{~m}, 5 \mathrm{H}, \mathrm{Ar}-\mathrm{H}), 6.95(\mathrm{~d}, J=8.8 \mathrm{~Hz}$, $1 \mathrm{H}, \mathrm{Py}-\mathrm{H}), 4.75$ (s, 2H, $\left.\mathrm{CH}_{2}\right), 4.34 \sim 4.48(\mathrm{~m}, 4 \mathrm{H}, 2 \times$ $\left.\mathrm{CH}_{2}\right), 2.62\left(\mathrm{~s}, 3 \mathrm{H}, \mathrm{SCH}_{3}\right) ;{ }^{13} \mathrm{C} \mathrm{NMR}\left(100 \mathrm{MHz}, \mathrm{CDCl}_{3}\right) \delta$ : $172.9,168.0,157.5,155.5,145.3,138.9,135.2,133.9$, $131.9,128.5,127.5,125.4,123.0,122.8,122.5,121.7$, $118.1,117.6,112.3,111.5,108.1,74.7,67.6,62.4,49.1$, 18.4. Anal. calcd for $\mathrm{C}_{26} \mathrm{H}_{21} \mathrm{Cl}_{2} \mathrm{~F}_{3} \mathrm{~N}_{4} \mathrm{O}_{3} \mathrm{~S}$ : C 52.27, H 3.54, $\mathrm{N}$ 9.38; found C 52.14, H 3.65, N 9.24.

2-氰基-3-甲硫基-3-[4-(5-三氟甲基吡啶-2-胺基)]苯 甲基胺基丙烯酸 $[2-(2,4-$ 二氯苯氧基)]乙酯(7i): 红色固 体, 产率 63\%. m.p. 124 $126{ }^{\circ} \mathrm{C} ;{ }^{1} \mathrm{H}$ NMR (400 MHz, DMSO-d $\left.d_{6}\right) \delta: 10.18(\mathrm{~s}, 1 \mathrm{H}, \mathrm{NH}), 9.67$ (s, 1H, NH), 8.48 (s, 1H, Py-H), $7.84 \sim 7.87$ (m, 1H, Py-H), 7.70 (d, $J=8.4$ Hz, 2H, Ar-H), 7.55 (d, $J=2.4 \mathrm{~Hz}, 1 \mathrm{H}, \mathrm{Ar}-\mathrm{H}), 7.22 \sim 7.36(\mathrm{~m}$, $4 \mathrm{H}, \mathrm{Ar}-\mathrm{H}), 6.95$ (d, $J=8.8 \mathrm{~Hz}, 1 \mathrm{H}, \mathrm{Py}-\mathrm{H}), 4.75$ (s, 2H, $\left.\mathrm{CH}_{2}\right), 4.46\left(\mathrm{t}, J=4.4 \mathrm{~Hz}, 2 \mathrm{H}, \mathrm{CH}_{2}\right), 4.32(\mathrm{t}, J=4.4 \mathrm{~Hz}$, $\left.2 \mathrm{H}, \mathrm{CH}_{2}\right), 2.62\left(\mathrm{~s}, 3 \mathrm{H}, \mathrm{SCH}_{3}\right) ;{ }^{13} \mathrm{C} \mathrm{NMR}(100 \mathrm{MHz}$, $\left.\mathrm{CDCl}_{3}\right) \delta: 172.9,168.0,157.7,153.0,146.0,139.1,134.9$, $131.6,130.0,128.5,127.7,126.6,125.5,124.9,124.3$, $122.8,121.4,118.1,117.7,115.5,107.9,74.6,67.7,62.5$, 49.2, 18.4. Anal. calcd for $\mathrm{C}_{26} \mathrm{H}_{21} \mathrm{Cl}_{2} \mathrm{~F}_{3} \mathrm{~N}_{4} \mathrm{O}_{3} \mathrm{~S}$ : C 52.27, $\mathrm{H}$
3.54, N 9.38; found C 52.40, H 3.42, N 9.50.

2-氰基-3-甲硫基-3-[4-(5-三氟甲基吡啶-2-胺基)]苯 甲基胺基丙烯酸 $[2-(3,4-$ 二氯苯氧基)]乙酯(7j): 红色固 体, 产率 $60 \%$. m.p. $142 \sim 144{ }^{\circ} \mathrm{C} ;{ }^{1} \mathrm{H}$ NMR (400 MHz, DMSO- $\left.d_{6}\right) \delta: 10.18(\mathrm{~s}, 1 \mathrm{H}, \mathrm{NH}), 9.67(\mathrm{~s}, 1 \mathrm{H}, \mathrm{NH}), 8.48(\mathrm{~s}$, 1H, Py-H), $7.84 \sim 7.87$ (m, 1H, Py-H), 7.70 (d, $J=8.4 \mathrm{~Hz}$, 2H, Ar-H), 7.50 (d, J=8.8 Hz, 1H, Ar-H), 6.94 7.27 (m, 5H, Ar-H and Py-H), 4.75 (s, 2H, $\left.\mathrm{CH}_{2}\right), 4.43$ (t, $J=4.0 \mathrm{~Hz}$, $\left.2 \mathrm{H}, \mathrm{CH}_{2}\right), 4.26\left(\mathrm{t}, J=4.0 \mathrm{~Hz}, 2 \mathrm{H}, \mathrm{CH}_{2}\right), 2.62(\mathrm{~s}, 3 \mathrm{H}$, $\left.\mathrm{SCH}_{3}\right) ;{ }^{13} \mathrm{C} \mathrm{NMR}\left(100 \mathrm{MHz}, \mathrm{CDCl}_{3}\right) \delta: 173.0,168.1$, $157.7,157.5,145.9,139.2,134.9,132.8,131.5,130.7$, $130.1,128.5,125.5,124.9,124.5,122.8,121.4,118.1$, $116.8,114.8,108.0,74.5,66.4,62.6,49.2$, 18.3. Anal. calcd for $\mathrm{C}_{26} \mathrm{H}_{21} \mathrm{Cl}_{2} \mathrm{~F}_{3} \mathrm{~N}_{4} \mathrm{O}_{3} \mathrm{~S}$ : C 52.27, $\mathrm{H}$ 3.54, N 9.38; found $\mathrm{C} 52.16, \mathrm{H} 3.43, \mathrm{~N} 9.52$.

2-氰基-3-甲硫基-3-[4-(5-三氟甲基吡啶-2-胺基)]苯 甲基胺基丙烯酸 $[2-(4$-溴苯氧基)]乙酯(7k): 红色固体, 产率 63\%. m.p. $138 \sim 139{ }^{\circ} \mathrm{C} ;{ }^{1} \mathrm{H}$ NMR $(400 \mathrm{MHz}$, DMSO- $\left.d_{6}\right) \delta: 10.19(\mathrm{~s}, 1 \mathrm{H}, \mathrm{NH}), 9.67$ (s, 1H, NH), 8.48 (s, $1 \mathrm{H}, \mathrm{Py}-\mathrm{H}), 7.85 \sim 7.87$ (m, 1H, Py-H), 7.70 (d, $J=8.4 \mathrm{~Hz}$, 2H, Ar-H), 7.44 (d, $J=9.2 \mathrm{~Hz}, 1 \mathrm{H}, \mathrm{Ar}-\mathrm{H}), 7.26$ (d, $J=8.4$ $\mathrm{Hz}, 2 \mathrm{H}, \mathrm{Ar}-\mathrm{H}), 6.93 \sim 6.96$ (m, 3H, Ar-H and Py-H), 4.75 (s, 2H, $\left.\mathrm{CH}_{2}\right), 4.42$ (t, $\left.J=4.4 \mathrm{~Hz}, 2 \mathrm{H}, \mathrm{CH}_{2}\right), 4.21$ (t, $J=4.4$ $\left.\mathrm{Hz}, 2 \mathrm{H}, \mathrm{CH}_{2}\right), 2.62\left(\mathrm{~s}, 3 \mathrm{H}, \mathrm{SCH}_{3}\right) ;{ }^{13} \mathrm{C} \mathrm{NMR}(100 \mathrm{MHz}$, $\left.\mathrm{CDCl}_{3}\right) \delta: 172.9,168.1,157.7,157.6,146.0,139.2,134.9$, $132.3,131.5,128.5,125.5,122.8,121.4,118.1,118.0$, 117.7, 116.7, 113.4, 107.9, 74.6, 66.1, 62.8, 49.2, 18.3. Anal. calcd for $\mathrm{C}_{26} \mathrm{H}_{22} \mathrm{BrF}_{3} \mathrm{~N}_{4} \mathrm{O}_{3} \mathrm{~S}$ : C 51.41, H 3.65, N 9.22; found $\mathrm{C} 51.55, \mathrm{H} 3.52, \mathrm{~N} 9.35$.

2-氰基-3-甲硫基-3-[4-(5-三氟甲基吡啶-2-胺基)]苯 甲基胺基丙烯酸(2-甲氧基)乙酯(7l): 红色固体, 产率 68\%, m.p. 108 $110{ }^{\circ} \mathrm{C} ;{ }^{1} \mathrm{H}$ NMR (400 MHz, $\mathrm{CDCl}_{3}$ ) $\delta$ : 10.33 (s, 1H, NH), 8.46 (s, 1H, Py-H), $7.68 \sim 7.70(\mathrm{~m}, 1 \mathrm{H}$, Py-H), 7.45 (d, J=8.0 Hz, 2H, Ar-H), $7.24 \sim 7.28$ (m, 2H, Ar-H), 6.86 (d, $J=8.0 \mathrm{~Hz}, 1 \mathrm{H}, \mathrm{Py}-\mathrm{H}), 4.77$ (d, $J=4.0 \mathrm{~Hz}$, $\left.2 \mathrm{H}, \mathrm{CH}_{2}\right), 4.32\left(\mathrm{t}, J=4.0 \mathrm{~Hz}, 2 \mathrm{H}, \mathrm{CH}_{2}\right), 3.67(\mathrm{t}, J=6.0 \mathrm{~Hz}$, $\left.2 \mathrm{H}, \mathrm{CH}_{2}\right), 3.41\left(\mathrm{~s}, 3 \mathrm{H}, \mathrm{OCH}_{3}\right), 2.69\left(\mathrm{~s}, 3 \mathrm{H}, \mathrm{SCH}_{3}\right) ;{ }^{13} \mathrm{C}$ NMR (100 MHz, $\left.\mathrm{CDCl}_{3}\right) \delta: 172.6,168.3,157.8,145.9$, $139.1,134.9,131.7,128.5,125.5,122.8,121.5,118.2$, 117.9, 117.6, 107.9, 74.9, 70.3, 63.9, 59.3, 49.1, 18.3. Anal. calcd for $\mathrm{C}_{21} \mathrm{H}_{21} \mathrm{~F}_{3} \mathrm{~N}_{4} \mathrm{O}_{3} \mathrm{~S}$ : C 54.07, $\mathrm{H}$ 4.54, N 12.01; found $\mathrm{C}$ 54.21, $\mathrm{H} 4.41, \mathrm{~N} 12.13$.

2-㲵基-3-甲硫基-3-[4-(5-三氟甲基吡啶-2-胺基)]苯 甲基胺基丙烯酸(2-乙氧基)乙酯(7m)：灰色固体，产率 $63 \%$, m.p. 109 $111{ }^{\circ} \mathrm{C} ;{ }^{1} \mathrm{H}$ NMR (400 MHz, DMSO- $d_{6}$ ) 
$\delta: 10.19$ (s, 1H, NH), 9.67 (s, 1H, NH), 8.48 (s, 1H, Py-H), $7.84 \sim 7.87(\mathrm{~m}, 1 \mathrm{H}, \mathrm{Py}-\mathrm{H}), 7.70(\mathrm{~d}, J=8.8 \mathrm{~Hz}, 2 \mathrm{H}, \mathrm{Ar}-\mathrm{H})$, $7.26(\mathrm{~d}, J=8.4 \mathrm{~Hz}, 2 \mathrm{H}, A r-\mathrm{H}), 6.94(\mathrm{~d}, J=8.8 \mathrm{~Hz}, 1 \mathrm{H}$, Py-H), 4.75 (d, $\left.J=4.8 \mathrm{~Hz}, 2 \mathrm{H}, \mathrm{CH}_{2}\right), 4.20$ (t, $J=4.8 \mathrm{~Hz}$, $2 \mathrm{H}, \mathrm{CH}_{2}$ ), $3.58\left(\mathrm{t}, J=4.8 \mathrm{~Hz}, 2 \mathrm{H}, \mathrm{CH}_{2}\right), 3.46(\mathrm{q}, J=7.2$ $\left.\mathrm{Hz}, 2 \mathrm{H}, \mathrm{CH}_{2}\right), 2.62\left(\mathrm{~s}, 3 \mathrm{H}, \mathrm{SCH}_{3}\right), 1.10(\mathrm{t}, J=7.2 \mathrm{~Hz}, 3 \mathrm{H}$, $\left.\mathrm{CH}_{3}\right) ;{ }^{13} \mathrm{C}$ NMR $\left(100 \mathrm{MHz}, \mathrm{CDCl}_{3}\right) \delta: 172.6,168.3,157.6$, $145.7,138.9,135.1,132.0,128.5,121.6,118.2,118.0$, 117.7, 107.9, 75.1, 68.1, 66.9, 64.1, 49.1, 18.3, 15.2. Anal. calcd for $\mathrm{C}_{22} \mathrm{H}_{23} \mathrm{~F}_{3} \mathrm{~N}_{4} \mathrm{O}_{3} \mathrm{~S}$ : C 54.99, H 4.82, N 11.66; found C 54.86, H 4.95, N 11.78 .

2-氧基-3-乙硫基-3-[4-(5-三氟甲基吡啶-2-胺基)]苯 甲基胺基丙烯酸 [2-(4-氟苯氧基)]乙酯(7n): 红色固体, 产率 59\%. m.p. $117 \sim 118{ }^{\circ} \mathrm{C}$; ${ }^{1} \mathrm{H}$ NMR $(400 \mathrm{MHz}$, DMSO- $\left.d_{6}\right) \delta: 10.30(\mathrm{~s}, 1 \mathrm{H}, \mathrm{NH}), 9.67(\mathrm{~s}, 1 \mathrm{H}, \mathrm{NH}), 8.48(\mathrm{~s}$, 1H, Py-H), $7.85 \sim 7.88$ (m, 1H, Py-H), 7.70 (d, $J=8.4$ Hz, 2H, ArH), 7.26 (d, $J=8.4 \mathrm{~Hz}, 2 \mathrm{H}, \operatorname{ArH}), 6.94 \sim 7.14(\mathrm{~m}$, $5 \mathrm{H}, \mathrm{ArH}$ and $\mathrm{Py}-\mathrm{H}), 4.78\left(\mathrm{~s}, 2 \mathrm{H}, \mathrm{CH}_{2}\right), 4.43$ (t, $J=4.4 \mathrm{~Hz}$, $\left.2 \mathrm{H}, \mathrm{CH}_{2}\right), 4.20\left(\mathrm{t}, J=4.4 \mathrm{~Hz}, 2 \mathrm{H}, \mathrm{CH}_{2}\right), 3.15$ (q, $J=7.2$ $\mathrm{Hz}, 2 \mathrm{H}, \mathrm{CH}_{2}$ ), 1.23 (t, $\left.J=7.2 \mathrm{~Hz}, 3 \mathrm{H}, \mathrm{CH}_{3}\right) ;{ }^{13} \mathrm{C} \mathrm{NMR}$ $\left(100 \mathrm{MHz}, \mathrm{CDCl}_{3}\right) \delta: 170.9,168.1,158.7,157.7,156.3$, $154.6,145.8,139.1,135.0,131.7,128.5,125.5,122.8$, 121.5, 118.2, 118.0, 117.7, 116.1, 116.0, 115.7, 107.9, 75.8, 66.6, 62.9, 49.2, 30.3, 15.0. Anal. calcd for $\mathrm{C}_{27} \mathrm{H}_{24} \mathrm{~F}_{4} \mathrm{~N}_{4} \mathrm{O}_{3} \mathrm{~S}$ : C 57.85, H 4.32, N 9.99; found C 57.72, $\mathrm{H} 4.46, \mathrm{~N} 9.87$.

\section{6 除草活性测试}

药剂配置: 对于化合物, 用分析天平 $(0.0001 \mathrm{~g})$ 称取 一定质量的原药, 用含 1\%吐温-80 乳化剂的 $N, N$-二甲基 甲酰胺(DMF)溶解配置成 $1.0 \%$ 母液, 然后用蒸馏水稀释 备用.

试验方法: 盆栽法(普筛), 苗前土壤处理和苗后茎 叶处理 ${ }^{[21]}$. 供试靶标为芥菜、繁缕和小㢣. 取内径 7.5 $\mathrm{cm}$ 花盆, 装复合土(菜园土/育苗基质, $V: V=1: 2$ )至 $3 / 4$ 处, 直接播种上述三种杂草靶标(芽率 $\geqslant 85 \%$ ), 覆土 $0.2 \mathrm{~cm}$, 待杂草长至 3 叶期左右进行苗后茎叶喷雾处理, 而苗前土壤处理则在喷药前一天进行播种覆土并加水 保持土壤湿润. 根据提供的样品量, 各化合物按照 1500 $\mathrm{g} \cdot \mathrm{ha}^{-1}$ 剂量在自动喷雾塔(型号: $3 \mathrm{WPSH}-700 \mathrm{E}$ )施药后, 待杂草叶面药晾干后移入室温培养, $35 \mathrm{~d}$ 后调查对杂草 的活性(\%). 对照药为 NK-9717 即 2-氧基-3-甲硫基-3-(2氯-5-吡啶)甲胺基丙烯酸乙氧乙酯.

辅助材料(Supporting Information) 化合物 $7 \mathbf{a} \sim 7 \mathrm{n}$ 的 ${ }^{1} \mathrm{H}$ NMR 和 ${ }^{13} \mathrm{C}$ NMR 图谱. 这些材料可以免费从本刊网 站(http://sioc-journal.cn/)上下载.

\section{References}

[1] Liu, X.; Huang, R. Q.; Cheng, M. R.; Zhao, Y. G.; Li, Z. G. Chem. J. Chin. Univ. 1999, 20, 1404 (in Chinese).

(刘昕, 黄润秋, 程慕如, 赵毅刚, 李在国, 高等学校化学学报, 1999, 20, 1404.)

[2] Zou, X. M.; Yu, L. M.; Gao, Y.; Shi, H. L.; Fei, J.; Liu, B.; Li, H. F.; Hu, F. Z.; Yang, H. Z. Chin. J. Org. Chem. 2006, 26, 337 (in Chinese).

(邹小毛，郁丽敏，高颖，施欢乐，裴江，刘斌，李慧芬，胡方中， 杨华铮，有机化学, 2006, 26, 337.)

[3] Zhang, H.; Li, X. Y.; Hu, D. Y.; Yang, S.; Fan, H. T.; Wei, X.; Song, B. A. Chin. J. Org. Chem. 2011, 31, 1419 (in Chinese). (章浩, 李向阳, 胡德禹, 杨松, 范会涛, 宋宝安, 有机化学, 2011, 31, 1419.)

[4] Zhong, S. H.; Wang, C. F.; Song, Q. X.; Fan, M. L.; Liu, B. Y.; Wei, D. M.; Liu, J. B. Chin. J. Org. Chem. 2014, 34, 2324 (in Chinese). (钟世华, 王春风, 宋青霞, 范明亮, 刘兵玉, 危冬梅, 刘建兵, 有机化学, 2014, 34, 2324.)

[5] Wu, S. S.; Miao, W. K.; Wang, T. T.; Fang, J. X. Chin. J. Org. Chem. 2015, 35, 1484 (in Chinese).

(吴珊珊, 苗文科, 王婷婷, 方建新, 有机化学, 2015, 35, 1484.)

[6] Sun, H. K.; Wang, Q. M.; Huang, R. Q.; Li, H.; Li, Y. H. J. Organomet. Chem. 2002, 655, 182.

[7] Song, B. A.; Zhang, H. P.; Wang, H.; Yang, S.; Jin, L. H.; Hu, D. Y.; Pang, L. L.; Xue, W. J. Agric. Food Chem. 2005, 53, 7886.

[8] Liu, Y. X.; Cao, B. L.; Li, Y. H.; Song, H. B.; Huang, R. Q.; Wang, Q. M. J. Agric. Food Chem. 2007, 55, 3011.

[9] Zhao, Q. Q.; Liu, S. H.; Li, Y. H.; Wang, Q. M. J. Agric. Food Chem. 2009, 57, 2849.

[10] Ouyang, G. P.; Cai, X. J.; Chen, Z.; Song, B. A.; Bhadury, P. S.; Yang, S.; Jin, L. H.; Xue, W.; Hu, D. Y.; Zeng, S. J. Agric. Food Chem. 2008, 56, 10160.

[11] Dai, H.; Liu, J. B.; Miao, W. K.; Wu, S. S.; Qin, X.; Zhang, X.; Wang, T. T.; Fang, J. X. Chin. J. Org. Chem. 2011, 31, 1662 (in Chinese).

(戴红, 刘建兵, 苗文科, 吴珊珊, 秦雪, 张欣, 王婷婷, 方建新, 有机化学, 2011, 31, 1662.)

[12] Dai, H.; Shi, L.; Zhang, H. J.; Li, Y. Q.; Fang, J. X.; Shi, Y. J. Chin. J. Org. Chem. 2012, 32, 1060 (in Chinese).

(戴红, 施否, 张海军, 李永强, 方建新, 石玉军, 有机化学, 2012, 32, 1060.)

[13] Wang, X.; Wang, C. Q.; Fu, C. R.; Zou, X. M. Chin. J. Org. Chem. 2015, 35, 92 (in Chinese).

(王金金，王朝强，傅翠蓉，邹小毛，有机化学，2015, 35, 92.)

[14] Dai, H.; Zhuang. H. Y.; Shi, L.; Li, G.; Zhang, H. J.; Fang, Y.; Dai, B. J. Chin. J. Org. Chem. 2015, 35, 2399 (in Chinese).

(戴红, 庄辉阳, 施否, 李刚, 张海军, 方源, 戴宝江, 有机化学, 2015, 35, 2399.)

[15] Mu, C.-W.; Qin, Z.-H. Modern Agrochem. 2003, 2, 1 (in Chinese). (慕长伟, 覃兆海, 现代农药, 2003, 2, 1.)

[16] Lahm, G. P.; Selby, T. P.; Freudenberger, J. H.; Stevenson, T. M.; Myers, B. J.; Seburyamo, G.; Simth, B. K.; Flexner, L.; Clark, C. E.; Cordova, D. Bioorg. Med. Chem. Lett. 2005, 15, 4898.

[17] Tian, Z. Z.; Shao, X. S.; Li, Z.; Qian, X. H.; Huang, Q. C. J. Agric. Food Chem. 2007, 55, 2288.

[18] Lu, S. Y.; Shao, X. S.; Li, Z.; Xu, Z. P.; Zhao, S. S.; Wu, Y. L.; Xu, X. Y. J. Agric. Food Chem. 2012, 60, 322.

[19] Selby, T. P.; Lahm, G. P.; Stevenson, T. M.; Hughes, K. A.; Cordova, D.; Annan, I. B.; Barry, J. D.; Benner, E. A.; Currie, M. J.; Pa- 
hutski, T. F. Bioorg. Med. Chem. Lett. 2013, 23, 6341.

[20] Wang, Q. M.; Sun, H. K.; Cao, H. Y.; Cheng, M. R.; Huang, R. Q. J. Agric. Food Chem. 2003, 51, 5030.

[21] Shi, Y. J.; Li, Y.; Fang, Y.; Chen, J.; Ye, L. Y.; Ge, S. S.; Dai, H.
Chin. J. Org. Chem. 2016, 36, 2472 (in Chinese).

(石玉军, 李阳, 方源, 陈佳, 叶林玉, 葛书山, 戴红, 有机化学, 2016, 36, 2472.)

(Zhao, C.) 